\title{
Computational thinking development and assessment through tabletop escape games
}

\author{
Divya Menon $^{1,2}$, Margarida Romero ${ }^{2}$, Thierry Viéville ${ }^{1,2}$ \\ ${ }^{1}$ Inria, Mnemosyne Research Team and Science Outreach divya.menon@etu.univ- \\ cotedazur.fr, thierry.vieville@inria.fr \\ ${ }^{2}$ Learning, INnovation and Education, Université Côte d'Azur \\ margarida.romero@unice.fr
}

\begin{abstract}
The development and assessment of computational thinking (CT) is garnering a lot of attention and research since the last decade. However, CT learning is perceived as a time-consuming and frustrating experience by many $\mathrm{K}-12$ and university students. Hence, educators are coming up with various methodologies to make CT both accessible and engaging for learners, thus leading to a spurt in various game-based learning (GBL) approaches in this field, ranging from board games to educational robotic games. This paper strives to evaluate tabletop escape games as a potential tool to develop CT as a competency among $K-12$ learners and the assessment of its effectiveness in achieving the learning outcomes. To do this, the authors conducted a literature review of existing papers on tabletop games that use the theme of an escape game to develop and assess $C T$ as a competency. Based on a systematic analysis of the existing literature, they conducted an analytical review of three papers on tabletop games in CT to identify the CT components being developed, the evaluation methods explained in them, the current limitations faced by the authors of these three papers and the possible ways to circumvent such limitations. This paper also covers the design aspects to be considered for the development of a CT-based educational escape game to support and evaluate this competency and its components.
\end{abstract}

Keywords: Game-Based Learning, Escape Game, Board Game, Tabletop Game, Computational Thinking, Competency Assessment

\section{Introduction}

Since the last decade, computational thinking (CT) is being increasingly identified as an important competency for learners of all ages, not just by educators but also by researchers and policymakers [1] [2]. Most of our everyday activities rely on digital technologies for efficient functioning; hence future professionals need to develop CT competency for their personal and professional activities. A growing corpus of research is being developed to define learning activities aimed at supporting its development and assessment [1] [3] [4]. From the present review of literature on CT, there appears to be no debate on the importance of integrating it with K-12 education and its relevance not just in the world of computer science but in our everyday lives. It can help improve the current test-driven educational model by transforming it into a competency-based educational model.

Though there appear to be a variety of definitions available among current literature on CT when applied to the context of K-12 education, there is a growing consensus on the CT definition provided by Cuny, Snyder, and Wing [5], who define it as "the thought processes 
involved in formulating problems and their solutions so that the solutions are represented in a form that can be effectively carried out by an information-processing agent" (p.1). The ISTE repository [6] elaborates on this further by defining CT as a problem-solving process that includes identifying the problem, selecting appropriate tools to solve the problem (computers or other tools), organising and analysing collected data logically, representing this data using models or simulations, identifying and experimenting with possible solutions and finding ways to automate them (through algorithmic thinking) for maximum efficiency and applying this knowledge to a variety of other problems. But when viewed as a competency to be developed, Shute, Sun and Asbell-Clarke [7] explain that "computational thinking skills include managing information effectively and efficiently with technologies in our data-driven era" (p.1).

Grover and Pea [8] suggest that CT is a competency that needs to be developed among all learners. They cite Wing et al. [9] and explain that "CT being viewed as at the core of all STEM disciplines, it appears that computing in K-12 is an idea whose time has come" (p.2). As a competency, CT is not just about learning to code using block and text-based programming. Students need to be empowered with the necessary competencies to resolve problems by thinking critically and creatively. Resnick [10] explains that their "educational mission is to engage students in thinking creatively, to encourage systematic thinking and working collaboratively - essential skills for everyone in today's society" (para 5). Hershkovitz et al. [11] expand on this further by proposing that providing learners with programming environments where they can use their creativity could help in fostering their overall learning as well. In this context, CT is being recognised as one of the most suitable competencies to be developed because it encompasses problem-solving, critical thinking and logical thinking. Hence, its advantages are not restricted to only those who wish to pursue a career related to information technology.

An uninitiated learner may find CT a complex competency to acquire, either because of a lack of motivation or due to ineffective learning strategies [18] [19] [20]. Despite its acknowledged importance to the present and future generations, this identification of a lack of interest among learners might be one of the reasons that has prompted various studies into efficient learning methods to develop CT competency. While there are three approaches to develop the CT competency, namely computer programming [10], educational robotics [13] and unplugged activities [12], educators and researchers are considering the use of different game-based learning (GBL) activities within these approaches to engage learners with CT. GBL refers to a learning approach in which gameplay is designed around definitive learning outcomes and ensures the integration of game mechanics with experiential learning. In certain contexts, GBL can support the development of competencies and learning objectives [21]. Currently, escape games are gaining popularity as a GBL and gamification tool due to their immersive and engaging nature. Hence, in this paper, the authors strived to evaluate the effectiveness of the tabletop version of escape games as a potential GBL activity to develop and assess CT as a competency.

\subsection{Research questions addressed within this paper}

In this paper, the authors conducted a literature review of existing papers (in English) on tabletop escape games that were used as a medium to develop and assess CT as a competency. The purpose of this paper is to address the following research questions:

- Currently, are there any papers on tabletop games based on the theme of an escape game that study the development and assessment of CT?

- What CT components do these tabletop escape games cover?

- What methods do the authors of these papers use to assess the development of CT among K-12 and university students?

- Are there features of tabletop escape games that could make them a potential method to develop and assess CT as a competency? 
- Are there any limitations to using tabletop escape games as a learning approach towards CT? If so, how can they be circumvented?

Before evaluating the potential of tabletop escape games to develop and assess CT competency, it is crucial to first understand its components and their definitions, which will be discussed in the next section.

\subsection{Common CT components and their definitions}

Today, CT is not restricted to the knowledge of or related to computers but includes some pertinent cognitive abilities that can be imparted through computational topics. This includes not only computer programming itself but as pointed out in the referred definitions of CT [5] [7], information coding and decoding, formal language (with respect to human language) understanding and mechanical processes (for example, parallel versus sequential processes and process complexity). The available literature on CT competency offers diverse opinions on what components constitute this broad subject. Brennan and Resnick [17] propose a three-dimensional CT framework comprising computational concepts (such as sequences, parallelism, conditionals, etc.); computational practices (such as debugging, reusing or sharing code, etc.); and computational perspectives (such as questioning or collaborating). According to Wing [12], CT covers five core aspects, including conditional logic, distributed processing, debugging, simulation and algorithm building. Denning [13] elaborates on this by referring to the computer as a tool rather than the object of study in computation and computation as an ever-changing way of representing information. $\mathrm{He}$ classifies the principles of computer science into computing, coordination, communication, recollection, automation, design and evaluation. When Papert [14] used the term 'computational thinking' in Mindstorms, he also referred to computers as "objects-to-thinkwith" that can be used in K-12 education (p.182) as an alternative to traditional learning environments, because they can engage learners, regardless of age, owing to their "universality" (p.8).

Based on Wing's definition of CT [12] and Papert's [14] materiality of "objects-tothink-with", Romero, Lepage and Lille [13] developed a six-component CT framework (CT6) including the identification of the problem (COMPO1 for component 1), organisation and modeling of the problem $(\mathrm{COMPO} 2)$, formal systems $\left(\mathrm{COMPO}^{1}\right)$ to refer to code, physical systems (COMPO4) to refer to the hardware and other tangible connected objects, devising a solution (COMPO5) and solution evaluation and iterative improvement (COMPO6).

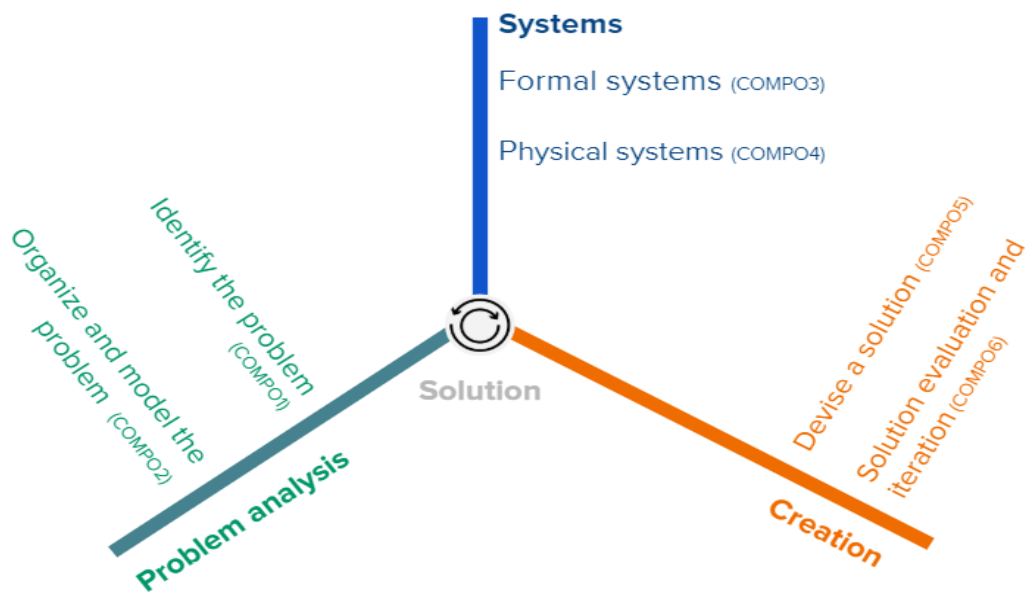

Figure 1: Computational thinking components of the CT6 framework [15]

\footnotetext{
${ }^{1}$ COMPO3 was initially named "code literacy" before being renamed as "formal systems"; COMPO4 was initially named "technology literacy" before being renamed as "physical systems" after a focus group on CT [19].
} 
The table below lists the CT components and subcomponents assessed under CT competency and their definitions, used as the coding schema for the analysis of tabletop escape games for $\mathrm{CT}$ in this paper.

Table 1: List of CT subcomponents in CT6 framework

\begin{tabular}{|c|c|c|}
\hline Categories & CT6 Components & CT6 Subcomponents and Definition \\
\hline \multirow{2}{*}{ Problem analysis } & $\begin{array}{l}\text { Identify the problem } \\
\text { (COMPO1) }\end{array}$ & $\begin{array}{l}\text { Analysing information and understanding the } \\
\text { problem and its components }\end{array}$ \\
\hline & $\begin{array}{l}\text { Organise and model the } \\
\text { problem (COMPO2) }\end{array}$ & Creating models based on possible solutions \\
\hline \multirow{9}{*}{ Systems } & \multirow{8}{*}{$\begin{array}{l}\text { Formal systems } \\
\text { (COMPO3) }\end{array}$} & $\begin{array}{l}\text { Sequencing - understanding the series of steps } \\
\text { for a task }\end{array}$ \\
\hline & & $\begin{array}{l}\text { Loops - figuring out ways to run the same steps } \\
\text { without repeating them from scratch }\end{array}$ \\
\hline & & $\begin{array}{l}\text { Parallelism - enabling processes/steps to run } \\
\text { simultaneously }\end{array}$ \\
\hline & & $\begin{array}{l}\text { Events - triggering something to happen based } \\
\text { on a previous step or action }\end{array}$ \\
\hline & & $\begin{array}{l}\text { Algorithms - describing them in simple tems } \\
\text { and understanding "blocks, objects, operators } \\
\text { and instructions" [34] }\end{array}$ \\
\hline & & $\begin{array}{l}\text { Conditionallogic - making decisions based on } \\
\text { if-then-else logic }\end{array}$ \\
\hline & & $\begin{array}{l}\text { Building algorithms - modelling a process at a } \\
\text { level where it can be processed by a machine }\end{array}$ \\
\hline & & Code optimisation - reusing and modifying code \\
\hline & $\begin{array}{l}\text { Physical systems } \\
\text { (COMPO4) }\end{array}$ & $\begin{array}{l}\text { Understanding features of the technology used } \\
\text { andits benefits and limitations }\end{array}$ \\
\hline \multirow{4}{*}{ Creation } & \multirow{3}{*}{$\begin{array}{l}\text { Devise a solution } \\
\text { (COMPO5) }\end{array}$} & $\begin{array}{l}\text { Identifying the programming features to be used } \\
\text { to accomplish a task }\end{array}$ \\
\hline & & $\begin{array}{l}\text { Using programming techniques to complete a } \\
\text { task }\end{array}$ \\
\hline & & Completing the programming task successfully \\
\hline & $\begin{array}{l}\text { Evaluate solutions and } \\
\text { conduct iterations } \\
\text { (COMPO6) }\end{array}$ & $\begin{array}{l}\text { Testing the solutions to see if they work; if they } \\
\text { don't work, analysing the reasons behind it and } \\
\text { finding solutions to problems that arise }\end{array}$ \\
\hline
\end{tabular}

The next section will introduce opportunities in GBL to support the development of different CT components and subcomponents.

\section{Educational escape games to develop CT}

Escape games originated as games played within a locked room where players work in teams to strategize their escape by solving puzzles and overcoming challenges to discover hidden keys. In recent years, they have gained tremendous popularity as a game-based and gamification tool that can be used to promote learning of any chosen subject for diverse groups of learners. Nicholson [16] defines escape games as "live-action team-based games where players discover clues, solve puzzles and accomplish tasks in one or more rooms in order to accomplish a specific goal (usually escaping from a room) in a limited time" (p.1). Today, educational escape games can be played in a classroom, online, through board games, or even from a box.

Escape games are based on a problem-solving narrative in which players are unaware of the expected outcomes. To play, they need to understand the problem and identify and implement a solution. Achieving the objectives of the escape game requires players to think 
creatively and work within their teams. Moreover, escape games could offer educators the flexibility to integrate any chosen subject with the theme of the game, which makes them suitable to accomplish any learning objectives, including those requiring a certain complexity such as in CT development. Educational escape games could be a potential medium to aid in developing and assessing CT components because they leverage the features of serious games to immerse learners in a narrative-based problem-solving scenario.

Educational escape games have the potential to be adapted to different disciplines in different learning environments. As a game that requires players to be actively involved in the problem-solving process, escape games could prove to be suitable for CT development and assessment. As with any competency, CT can also be effectively imbibed when learners practice the knowledge they gain. In escape games, learners are engaged to apply the knowledge they gain to complete challenges or tasks, thereby learning by doing [17]. They could offer learners the opportunity to experience, reflect on and apply the learning they gain during the course of a game [18]. Apart from being actively involved, learners should receive timely feedback on their progress and get ample opportunities for trial and error before they succeed in achieving the learning objectives without fear of failure [19]. This aspect makes escape games suitable, because irrespective of the medium in which learners play it, they apply their learning to accomplish tasks and hence, receive feedback in terms of visual representations or debriefing at the end of each task. Moreover, topics like CT may have a better chance of development among learners if a scaffolding approach is used. These games require learners to use their existing knowledge to complete challenges, thereby acquiring new knowledge along the way. Escape games usually have levels of progression which can be used by educators to initially introduce learners to foundational CT concepts. Gradually, as they progress to higher levels, learners can be provided with opportunities to gain advanced knowledge and apply it either during gameplay or through game creation.

Escape games are a specific subtype of cooperative games wherein players "work together to win or lose as a team" [20, p.2]. These games are based on positive interdependence [21], but they have specific features, such as the operationalisation through a narrative of space-time in which the character should escape a certain threat. The engagement of learners through escape games could be an interesting way of physically and cognitively engaging them, and similar to other cooperative games utilising positive interdependence to solve a complex task. Another specificity of escape games is their materiality and embodiment of the learner-player. While some cooperative games in sports education are based on embodiment and materiality (objects mediating the interactions), the embodiment and materiality for CT learning through escape games lead to a very specific learning activity engaging the learner in a multi-sensorial way of learning.

Board games are described as a medium to support and evaluate the players' CT components. In this paper, we will be evaluating educational escape games with reference to the CT components described by Romero, Lepage and Lille [15]. According to Berland and Lee [22], board games can help with this analysis better than digital games since in the digital version the game rules are strictly enforced by game mechanics, whereas in board and other tabletop games players are required to plan and execute strategies based on their knowledge of the game rules, thereby resorting to CT. They conducted a study on three groups of novice players (aged 17 to 19 years) using Pandemic, a strategy-based board game, in which players have to fight against four highly infectious diseases that are spreading in various cities across the world by collecting, sharing and using the information to ultimately win the game ${ }^{2}$. They analysed the responses of players in the five elements of procedural $\mathrm{CT}$, which include conditional action (deciding on the best moves within the game), algorithm building (devising a plan-of-action, which can be reused later for other moves), debugging (identifying errors based on moves taken and their impact on the game), simulation (using hypotheses to test possible outcomes) and distributed computing

${ }^{2}$ We invite the reader to play Pandemic game here: http://pandemic2.org/ 
(information sharing by different players while strategizing based on a rule-based plan). Berland and Lee [22] ended the paper on the note that while board games could positively contribute by helping players to develop CT components, further research was needed in this area.

In another example, Apostolellis et al. [23] devised a board game, RaBit EscAPE, with the primary objective of promoting CT through collaborative play for players between the ages of 6 to 10 years. In an informal study conducted using this board game, two groups of three players each aged 8 to 10 years from an elementary school were asked to combine wooden bits (that comprise varying magnet placements and polarity combinations) to first construct blocks and then paths over a predefined route to help the token (a rabbit) escape from the apes (bits that need to be repelled from the path). In this way, players are challenged to use logical thinking to analyse and organise data, algorithmic thinking to come up with the steps to be followed, simulation to identify and experiment with possible solutions, debugging when a possible solution doesn't work, as well as problem-solving and collaborative thinking, among other social competencies. The RaBit EscAPE game covers the problem analysis component of CT, which includes problem identification, organisation and modelling. It also makes players use sequencing, conditional logic and algorithm building skills that come under formal systems and the solution evaluation and iteration component. In the game, players compete against each other by starting on the opposite sides of the board to complete the path. By rolling the dice, they decide how many wooden bits they can use in each move to build their path. They need to strategize and hypothesize, thus using model building and simulation skills, which are components covered under CT. The game uses a board game without any digital devices; hence it doesn't cover the physical systems' component. Another important aspect to be considered is that since the writers conducted an exploratory study, the paper doesn't explain if the competencies mobilised by the players converted into code literacy for them. The study of Apostolellis et al. [23] doesn't address CT assessment explicitly, but their study engages learners in a tabletop escape game that covers most of the CT components, including logical thinking, algorithmic thinking, simulation, debugging, problem-solving and collaborative thinking. They concluded that there is a need to further develop the evaluation of their unplugged board game activity to understand its impact better.

Young learners can also be motivated to learn computer programming, and therefore, $\mathrm{CT}$, by using programming as a medium to integrate physical and cognitive activity, one that requires learners to use their hands and mind to achieve the given objectives [24]. A study was conducted in China on seven children aged around 8 years with some prior knowledge of computers but without any prior programming experience to test out this idea. They used T-Maze, a physical programming tool for children between the ages of 5 to 9 years, to play multi-level maze escape games and build their own mazes by tinkering with some wooden blocks. This game seems to be inspired by the concept of board games as well as escape games. They connected wooden blocks, some of which are equipped with magnets and others with sensors, to either move a virtual avatar on the screen to escape from a maze (using sensor blocks) or build a new maze by recreating a maze map and passable paths. TopCode [25], a computer vision system, was used in the study to identify symbols on blocks to convert physical programs into digital codes automatically. During the play, children received real-time visual and audio feedback, which helped them analyse and debug their programs. In T-Maze there was no time pressure on learners to complete tasks. With this study, they concluded that children were able to learn concepts of abstraction, automation (loops), problem decomposition and analysis and creativity. The authors also specified how children were able to create their own programs, achieve a high level of engagement and that there was a possibility that this game could help enhance children's understanding of CT.

Some researchers rely on digital versions of escape games to develop CT. For example, Program Your Robot [26] introduces learners to introductory concepts of CT, while developing relevant competencies through a digital puzzle-solving game. Players aged 17 to 19 years have to program a virtual robot by following a set of rules and using 
programming methods, such as sequence, conditionals, iteration and loops. They use a progressive learning method, in which each level introduces players to an advanced level of knowledge about CT competency. Though the writers are yet to conduct a formal study of this game, they have collected initial feedback from computer science students with prior knowledge of programming. According to Kazimoglu et al. [26], students found this game suitable to help them understand introductory programming concepts and they mention that "this type of gameplay allows players to visualise how programming constructs work" (p.7).

\subsection{Educational escape games to assess CT}

The previous section mentions various initiatives that support CT development through educational escape games. However, there is also a need for formative and summative assessment of CT that complements learner activities. Since escape games are usually implemented through diverse technologies and meant to be completed within a specific duration, there could be a variety of possibilities for learning game analytics in CT. Steiner et al. [27] cite the Society for Learning Analytics Research (SoLAR) when defining learning analytics as "the measurement, collection, analysis and reporting of data" (p.1). To collect this data, they classify learning games assessment into real-time game analytics and post-game analytics which help in "linking observable game behaviour with...learning outcomes and competence..." (p.2). In real-time game analytics, the assessment method is embedded within the game design by taking into account criteria, such as level progression, number of attempts, scores earned by a player and player inputs including the time to start and end the game and clicks or movements to accomplish a task. Post-game analytics include correlation of learning objectives with the behaviours and actions of players, as well as using post-tests and player feedback to collect and measure data. In escape games, learning data could be collected through observations during each play activity, by analysing video recordings or manual logs of player behaviour and actions (using specific coding schemas of the CT aspects to assess), or even pre-task or post-task questionnaires on CT competency, such as the Computational Thinking Test (CTT) [28]. In all these cases, there is a need to design the assessment of CT at the instructional level to ensure that it supports CT development in learners. Additionally, it could also be helpful for educators to be able to certify the achievement of certain learning objectives in relation to CT. We will be discussing different assessment methods used by some tabletop educational escape games in the fifth section of this paper.

\section{Methodology}

The objective of this study is to carry out a systematic analysis of specific components and subcomponents of CT supported through tabletop escape games. We have reviewed various published works in English on educational escape games that aim to develop CT competency. The literature references have been accessed using keywords that comprise escape games, board games, tabletop games and game-based learning for computational thinking. The primary sources for these papers are ResearchGate, Google Scholar and ScienceDirect. Though we haven't restricted the papers by their year of publication, special emphasis was given to review papers that covered educational escape games for learners of diverse age groups, with varying levels of knowledge in computer science and using different gaming and learning approaches to introduce CT. The different mediums used to implement these games include unplugged board games, escape games with computer programming and escape games using robotic tools. 


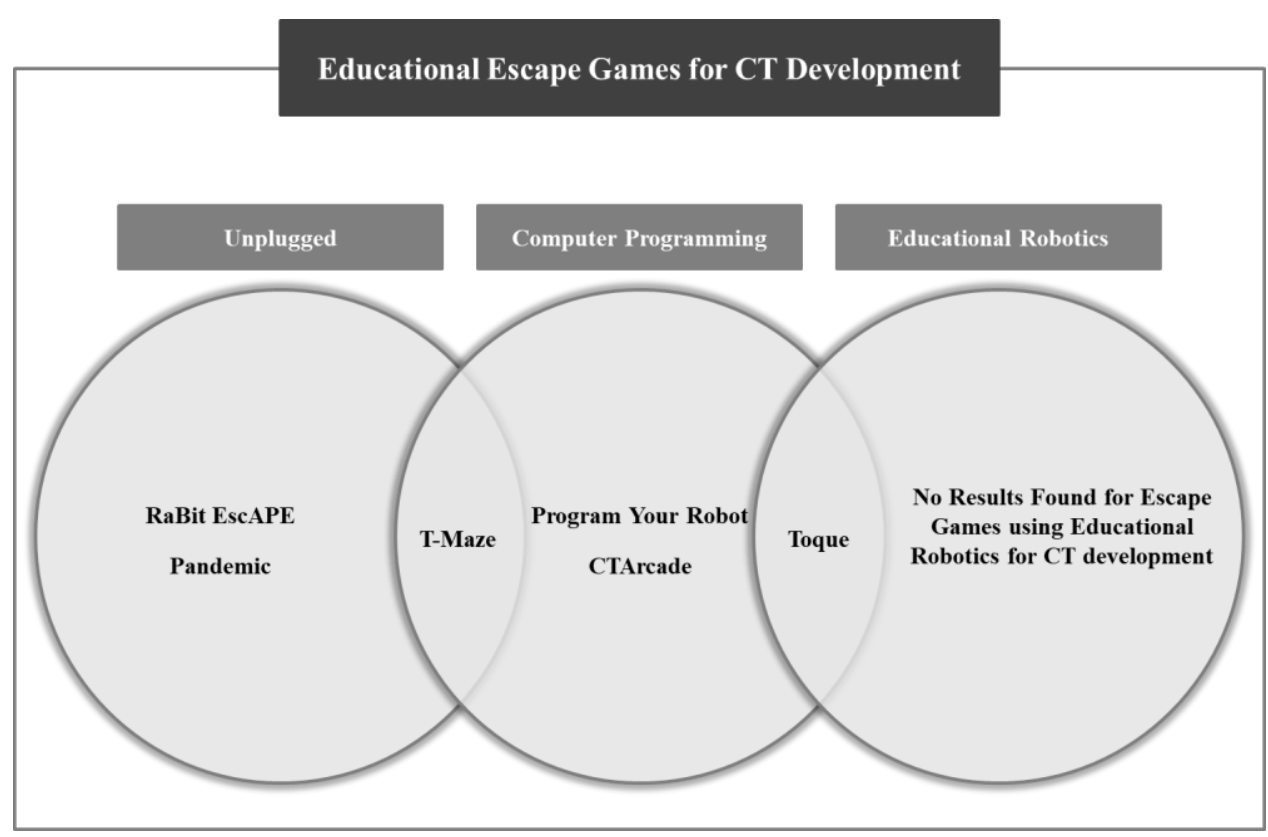

*No published papers with escape games using educational robotics to develop CT were found.

Figure 2: Mediums used by various educational escape games for CT

Another important criterion was to consider the CT components that were being covered through each of these games. In some of the papers, the writers mentioned the assessment of creativity, collaboration and CT among other competencies. Though we do not dispute their importance as critical competencies, we have focused on papers that target the achievement of these competencies by covering the main CT components.

At the initial stage, we began by searching for English papers on educational escape games, with a special focus on digital or tabletop games for CT development. We selected papers which focused on studies conducted for CT development in K-12 education (for learners between the ages of 6 to 19 years). A list of all the reviewed games and reasons for eliminating them from the purview of this literature review is mentioned in Table 2 of this paper.

Table 2: List of papers analysed for this paper

\begin{tabular}{|c|c|c|c|}
\hline S\# & Game Name & Topic & Reasons for selection or elimination \\
\hline 1 & Crabs and Turtles [29] & $\mathrm{CT}$ & $\begin{array}{l}\text { - Not based on escape game theme } \\
\text { - Game only tested for user experience so far }\end{array}$ \\
\hline 2 & CTArcade [30] & CT & $\begin{array}{l}\text { - Not based on escape game theme } \\
\text { - No observable behaviours explained }\end{array}$ \\
\hline 3 & $\begin{array}{l}\text { EleMental: The Recurrence } \\
{[31]}\end{array}$ & Computer programming & $\begin{array}{l}\text { - Not based on escape game theme } \\
\text { - Doesn't cover all CT components }\end{array}$ \\
\hline 4 & Escape Game [32] & Nursing protocols & - Not for CT development \\
\hline 5 & EscapED [33] & GBL for higher ed & - Not for CT development \\
\hline 6 & Escape Room Activity [34] & Computer Science & - Not for CT development \\
\hline 7 & Pandemic [22] & CT & $\delta$ This study meets all the selection criteria \\
\hline 8 & Program Your Robot [26] & CT & $\begin{array}{l}\text { - Not based on escape game theme } \\
\text { - Game only tested for user experience so far }\end{array}$ \\
\hline 9 & RaBit EscApe [23] & $\mathrm{CT}$ & This study meets all the selection criteria \\
\hline 10 & $T$-Mase $[24]$ & $\mathrm{CT}$ & $d$ This study meets all the selection criteria \\
\hline 11 & Toque [35] & Computer programming & $\begin{array}{l}\text { Not based on escape game theme } \\
\text { - Game only tested for user experience so far } \\
\text { - Doesn't cover all CT components }\end{array}$ \\
\hline
\end{tabular}


From this list, we shortlisted eight papers that use the concept of escape games for CT. Out of these, we filtered and shortlisted three tabletop games which include the RaBit EscAPE game [23], Pandemic board game [22] and T-Maze [24] based on the characteristics of escape games defined by Nicholson [16] and Clarke et al. [33] shown in Table 3.

Table 3: Checklist for Educational Escape Game Characteristics

\begin{tabular}{|r|l|c|c|c|}
\hline S\# & \multicolumn{1}{|c|}{ Characteristic } & RaBit EscApe & Pandemic & T-Maze \\
\hline 1 & Game based on a dventure or fantasy & $\checkmark$ & $\checkmark$ & $\checkmark$ \\
\hline 2 & Based on theme of escape or rescue & $\checkmark$ & $\checkmark$ & $\checkmark$ \\
\hline 3 & $\begin{array}{l}\text { Solving challenges/puzzles using resources within } \\
\text { game }\end{array}$ & $\checkmark$ & $\checkmark$ & $\checkmark$ \\
\hline 4 & Specific time limit & $\checkmark$ & $\checkmark$ & $\times$ \\
\hline 5 & Team-based & $\checkmark$ & $\checkmark$ & $\times$ \\
\hline 6 & Cooperative rather than competitive & $\checkmark$ & $\checkmark$ & $\checkmark$ \\
\hline 7 & Accomplishing a specific goal of escape or rescue & $\checkmark$ & $\checkmark$ & $\checkmark$ \\
\hline 8 & Strategizing moves that impact game outcomes & $\checkmark$ & $\checkmark$ & $\checkmark$ \\
\hline 9 & Leam by doing & & & $\checkmark$ \\
\hline
\end{tabular}

Each of these three games meets six or more characteristics of an educational escape game, as presented in Table 3. For example, in the RaBit EscApe game [23], Apostolellis et al. used the theme of helping rabbit tokens escape from enemy ape tokens to engage learners with the CT topic. Though Pandemic has not been proposed as an escape game by Berland and Lee [22], we have included it in our literature review for this paper since it also meets most of the requirements of an escape game. In their paper, the writers are studying whether this tabletop game can help in the development of CT spontaneously during gameplay. It is a collaborative game in which players need to escape from and avoid the spreading of viruses into cities within the game by strategizing and planning their moves. Similar to escape games, Pandemic is based on a problem scenario that players need to solve by cooperating rather than playing against each other within a stipulated time (before the four diseases spread to all the cities). During the game, additional challenges are introduced and players are expected to solve them by sharing knowledge and discovering additional strategies. Their actions impact the outcomes at every stage of the game and the overall game outcomes as well. Lastly, the T-Maze [24] game also uses the concept of escaping from a maze.

These games were shortlisted based on the information provided in these papers about the methodology through which they identified observable behaviours to ensure CT components were introduced among their players. The remaining five papers were excluded from this literature review since they propose an educational escape game that could be used to develop CT, however, have not yet conducted any studies, formal or otherwise, in which the game was played by learners and observed for its effectiveness as a possible learning tool for CT. Even though only three papers meet the selection criteria, the quality of the studies, the diversity of game types chosen (Pandemic, RaBit EscApe and T-Maze) and the level of the description provided us with ample information to achieve the research objectives of our study.

\subsection{Instruments and Coding}

The systematic analysis of the shortlisted tabletop escape game papers for CT development was conducted based on the components introduced in the theoretical framework. Three researchers, with an expertise in CT assessment, worked together to evaluate these papers 
pag. 12

and discuss the ambiguities that appeared during the analysis process. While we have not replicated the methodology of these games, we have analysed the methodology and results of these studies. This analysis was developed using a framework of six CT components based on Wing's [5] definition of CT.

\section{Results}

The table below is organised according to the CT components being analysed. Coloured cells indicate those components which were not only defined as a learning objective but also operationalised as observable behaviours to be evaluated in each of the abovementioned three games.

Table 4: Systematic analysis of the three tabletop escape games for CT development

\begin{tabular}{l|l|l|l}
\hline \multicolumn{1}{c|}{ Subcomponents assessed } & RaBit EscAPE & Pandemic & T-Maze \\
\hline Problem identification & & & \\
\hline Problem organisation and modelling & & & \\
\hline Sequencing & & & \\
\hline Loops & & & \\
\hline Parallelism & & & \\
\hline Events & & & \\
\hline Understanding algorithms & & & \\
\hline Conditionallogic & & & \\
\hline Building algorithms & & & \\
\hline Code optimisation & & & \\
\hline Understanding physical systems & & \\
\hline Identification of programming features & & & \\
\hline Usage of programming techniques & & & \\
\hline Completing programming tasks & & & \\
\hline Solution evaluation and conductingiterations & & & \\
\hline
\end{tabular}

In the three games analysed in terms of the operationalisation of CT competency in this paper, the writers have specified that they are yet to conduct a formal study to assess CT development. However, they have identified what observable indicators will help in the assessment stage when an empirical study is conducted.

From our analysis, we can observe that all the three games cover most of the CT components, including all the components under the problem analysis axis as well as under the creation axis. Under the systems axis, most of the games didn't provide learners with the opportunity to gain an understanding of the physical systems. Moreover, all three games required players to use the concept of algorithms to achieve an objective, but the players didn't understand that they were using algorithms. Writing codes was not accomplished in any of the games. As specified clearly in the three papers, though the players used specific 
CT components to achieve success in the games, those components didn't transform into an understanding of the underlying concepts that are part of the CT competency. Next, we will provide references from each of the papers about these assessment indicators for each of the subcomponents.

In the RaBit EscAPE game, the writers conducted an informal study using an unplugged approach to develop CT competency. Based on their learning from this initial study, they have given examples of how they will assess the CT competency marked in Table 2 in future studies conducted on this game. The observable behaviours are mentioned on page four of their paper. To assess model formation and simulation, players should be able to "form and contrast hypotheses" and "draw the blocks on the board but without placing the bits". Conditional logic will be identified when players analyse polarity of the magnets on each wooden block in response to the path to be created while repelling the ape blocks based on the minus and plus signs (for example, "If I place this bit here, then this block has a minus on the bottom and needs a big square piece with a plus on the top"). This will also help players practice and understand pattern recognition when "identifying which bits create blocks that can fit the path printed on the board while also attracting adjacent blocks or repelling ape blocks". Players should be able to implement algorithm building by "combining different requirements to build a set of steps that will lead to an efficient solution of a problem". To logically organise and analyse data, players will have to arrange the wooden pieces into blocks first and use block combinations that correspond to the path to be built. Finally, though players won't receive real-time feedback, they'll have to wait till they finish building the path to attempt iteration and debugging (i.e., "in case of wrong placement of bits, starting all over to complete the path in the correct way").

In the paper on the Pandemic game, the writers rely on the CT definitions provided by Wing [5], Papert [14] and National Research Council [36] and also use an unplugged board game for their study. As they explain in the paper, they use the players' conversations and behaviours to systematically analyse whether the CT components were developed through this game. Three teams of players were asked to play the game separately and played within 60 to 90 minutes. To $\log$ the data for analysis, the players' activities were video recorded and transcripts were generated from these videos. The videos were first classified according to gameplay turns and then subdivided based on utterances used by players. The utterances were then translated into rule comprehension and strategies on the part of the players. Based on this interpretative analysis, the writers created CT-related codes that are being cited from page nine of this paper. Players practised conditional logic when they had to "describe the chain of events that might happen based on the game's rules, should a particular action be taken". When the "player who moves their token to various spots and declares the actions they would take without releasing the token (and thus committing herself to a set of decisions)" (p. 9) they were simulating and modelling possible strategies. By clarifying the rules or strategies to be used in the game, the players resorted to the debugging method. Formulating a plan of action helped the players build an algorithm that can be generalised and used for future events within the game. The writers claim that the purpose of this paper was to demonstrate how computational thinking occurs through the gameplay of a strategic board game. However, what appears to be lacking in the paper is the comprehension of CT subcomponents among players, without focusing on the assessment of these subcomponents.

The T-Maze game [24] uses a tangible programming approach. The writers conducted a study involving 20 children in a lab environment to improve CT in "children's programming activities" (p.5). Two kinds of activities in the study included gameplay using maze escape and game programming using maze creation. The paper describes how each CT component development was observed during the game on page five of the paper. The players begin by identifying the problem by "knowing the ports where the path starts and ends, ...connect them ...with programming blocks". They engage in organising and modelling the solution by comparing their plans to select the shortest path. Simulation can be observed when "children need to think about how to perceive the world coordinates of the virtual maze and how to map the behaviours in real world". After the simulation, players 
have to "map themselves into the virtual characters on screen and control the characters' behaviours" thereby implementing abstraction. The players engage in using formal systems through automation when they program using a run loop which "updates the feedback on screen and interacts with users based on designed rules for sensors" to complete the escape from the maze. Finally, they use real-time feedback to test and debug their programs.

\section{Discussion}

Several attempts are being made to use escape games in CT competency development across the globe, however, in most cases, there is a lack of published literature on the activity, its evaluation methods and the data to support its effectiveness. For example, Webby's Challenge is an escape room concept developed by Frey [37] in the USA to introduce $\mathrm{CT}$ and coding activities to learners aged 10 years and above. Though the website introduces and explains the method of implementing this game in a classroom, the research data and learning game logs to support its effectiveness are missing. Among the escape games published in gaming platforms, Escape Code - Coding Adventure on Steam is a digital escape game that claims to integrate the subjects of coding, technology and artificial intelligence to allow players to learn while playing. However, it doesn't provide any description of the method being employed or about its effectiveness in achieving the learning objectives [38].

Escape games are primarily regarded as activities that require special infrastructure and additional resources, including a room dedicated for the game, designers and facilitators, time and money to develop and evaluate the game and its effectiveness. These requirements might dissuade many educators from integrating escape games in their learning practices. Using miniature representations of escape games, such as Breakout EDU kits [39], is a great idea, however, the cost of such kits could be a major concern for educational organisations from economically marginalised countries. A welcome approach would be to use tabletop escape games that can be created with a do-it-yourself approach which would encourage educators and learners to create their own escape games. Nevertheless, the design of a CT escape game would require them to follow a method of introducing, developing and evaluating CT components.

One of the biggest limitations in the implementation of CT development in K-12 education is in choosing the most effective medium of assessment, and this becomes more challenging with escape games. Werneburg et al. [40] refer to the assessment method used by Brennan and Resnick [41] who performed interviews to evaluate aspects like idea development and debugging of an activity based on their belief that CT cannot be assessed from a binary state of yes or no in terms of whether a task has been accomplished or not. They also mention the use of questionnaires by Grover and Basu [42] to detect errors in an activity, but the presence of errors in a programming syntax does not necessarily denote the lack of the CT competency. Romero et al. [15] have identified three prevalent approaches while assessing CT. The first type of assessment is followed by the Computer Science Teachers Association's curriculum in the USA, wherein the content progresses based on grade, from low-level programming to object-oriented programming, but doesn't take into account competencies such as creativity. The second approach is as per the Barefoot's CT model in the UK which includes assessing the learners' ability to demonstrate logic, algorithms, decomposition, patterns, abstraction and evaluation, but it doesn't evaluate knowledge retention after the assessment. The third approach is the kind that is followed by $D r$. Scratch [43], which automatically scores a Scratch program on aspects, such as abstraction and logical thinking. However, as discussed before, though Dr. Scratch provides feedback on how a program can be improved, it does not take into account the objective that the learner sets out to accomplish or "the task demanded" (p.6). In many of the examples reviewed in this paper, there was a lack of information on the logs or how the assessment of CT components was done through these games. Some games, such as the 
RaBit EscAPE game [23], aim to develop multiple competencies ranging from problemsolving to efficiency, however, how the logs are developed are not clearly explained. When using a strategic board game, such as Pandemic [22] to assess the CT competency development, logs were maintained in the form of videos and transcripts to evaluate gameturns and utterances between players and translating these into codes for the strategies devised by players. But further research is needed to evaluate whether such games can support the development of CT competency.

Studies are being conducted to identify suitable assessment models and tools for CT concepts. In their paper, Romero et al. [15] conducted a study with 120 undergraduate students at Université Laval in Canada to participate in a story2code creative programming challenge using Scratch and used two tools to conduct their assessment, including Dr. Scratch and \#5c21, which is a tool developed by them wherein an educator identifies observable states with four levels of achievement for each activity. While Dr. Scratch is automated, $\# 5 \mathrm{c} 21$ requires human expertise of the subject, and both tools assess different skills. Though they were able to successfully log the achievement levels of students through this study, the authors concluded by stating that this approach needs to be further developed before it can be used to evaluate complex activities aimed at developing the CT competency. It can also be noticed that nowadays the CT components on formal systems mainly focus on sequential imperative programming (at the difference of collective protocols or other programming paradigms such as functional programming) and only consider basic scalar value or list. Taking into account more complex useful data structures, other information coding issues, or other computational science mechanisms, such as those used in machine learning (often called artificial intelligence) is also useful. In both the educational science challenges and the actual curricula, it is reasonable to restrain to the former domain of sequential imperative programming with basic data structures, but we can be fairly certain that more issues are going to emerge in the near future, which necessitates further research on how such competencies can be learned. Through escape games, CT assessment can be developed through an engaging activity for learners in which the embodiment is higher than other survey-based assessments [44].

To ensure that an escape game could help achieve the expected learning outcomes, there are a few other important points to keep in mind as well. For this objective, we can start by identifying target players for whom the game is being designed. It is also important to identify topics and competencies to be covered and the learning objectives supporting the game activity within a perspective of constructive alignment [53] [54]. An engaging escape game needs to have a clear problem-solving objective and be based on a storyline that sets the tone and theme for the activity. Since time pressure could hamper the learning process, designers and educators need to decide on using it minimally or avoid it altogether to ensure a positive learning experience for players. The game design of escape games can consider the age and prior knowledge of target players to correctly adapt the GBL activities. Based on the existing research on game mechanics [40] [55] we can consider real-time feedback mechanisms to keep players engaged in the learning process and to enable them to debug their methods within the time constraints of the game. However, further research should be developed to better understand the way to consider time pressure and constraints in a way to support engagement without hindering the learning process. Debriefing at the end of every level or game must be integrated when designing the game to ensure that learners understand the concepts and knowledge retention is successfully achieved [25].

\section{Conclusion}

Through the systematic analysis of the literature on tabletop escape games for CT competency development, the authors observe the potential of these games to engage and sustain a learner's interest in the game while they develop CT competency. Through a detailed analysis of three tabletop escape games for CT development, it is observed that 
pag. 16

both the unplugged activities approach and the computer programming approach could help the learners remain actively involved in the learning process. Since escape games encourage players to use their problem-solving and cognitive abilities, they can be used as serious games for CT. Keeping in mind the different limitations that escape rooms might have when used for educational purposes in diverse educational backgrounds, tabletop escape games could be a possible solution to ensure learner engagement in the learning activity. However, a detailed review of these papers also highlights that the assessment methods to evaluate the effectiveness of these games need to be developed and tested in a more precise manner. The authors of this paper propose that further research is needed to ascertain how tabletop escape games can help in achieving the learning outcome of developing CT as a competency among K-12 learners.

\section{References}

[1] A. Yadav, C. Mayfield, N. Zhou, S. Hambrusch, and J. T. Korb, "Computational thinking in elementary and secondary teacher education," ACM Trans. Comput. Educ. TOCE, vol. 14, no. 1, p. 5, 2014. https://doi.org/10.1145/2576872

[2] V. Barr and C. Stephenson, "Bringing computational thinking to K-12: what is Involved and what is the role of the computer science education community?," ACM Inroads, vol. 2, no. 1, pp. 48-54, 2011. https://doi.org/10.1145/1929887.1929905

[3] P. Curzon, M. Dorling, T. Ng, C. Selby, and J. Woollard, "Developing computational thinking in the classroom: a framework," 2014.

[4] S. Y. Lye and J. H. L. Koh, "Review on teaching and learning of computational thinking through programming: What is next for K-12?," Comput. Hum. Behav., vol. 41, pp. 51-61, 2014. https://doi.org/10.1016/j.chb.2014.09.012

[5] J. Wing, L. Snyder, and J. M. Wing, "(PDF) Computational Thinking: What and Why?" 2010.

[6] ISTE, "Computational Thinking Leadership Toolkit," 2015. [Online]. Available: https://id.iste.org/docs/ct-documents/ct-leadershipt-toolkit.pdf. [Accessed: 05-Apr-2019].

[7] V. J. Shute, C. Sun, and J. Asbell-Clarke, "Demystifying computational thinking," Educ. Res. Rev., vol. 22, pp. 142-158, 2017. https://doi.org/10.1016/j.edurev.2017.09.003

[8] S. Grover and R. Pea, "Computational Thinking in K-12 A Review of the State of the Field," Educ. Res., vol. 42, no. 1, pp. 38-43, Jan. 2013. https://doi.org/10.3102/0013189X12463051

[9] J. Wing, P. Henderson, O. Hazzan, and T. Cortina, "• An Overview of Computational Thinking," p. 7, 2007. https://doi.org/10.1145/1227310.1227378

[10] M. Resnick, "A Different Approach To Coding - BRIGHT Magazine," Medium, 26-Jun-2019. [Online]. Available: https://brightthemag.com/a-different-approach-to-coding-d679b06d83a. [Accessed: 16-Jul-2019].

[11] A. Hershkovitz, R. Sitman, R. Israel-Fishelson, A. Eguíluz, P. Garaizar, and M. Guenaga, "Creativity in the acquisition of computational thinking," Interact. Learn. Environ., pp. 1-17, 2019.

[12] J. M. Wing, "Computational thinking," Commun. ACM, vol. 49, no. 3, pp. 33-35, 2006. https://doi.org/10.1145/1118178.1118215

[13] P. J. Denning, "The Profession of IT Beyond Computational thinking," p. 3, 2009. https://doi.org/10.1145/1516046.1516054

[14] S. Papert, Mindstorms: children, computers, and powerful ideas. New York: Basic Books, 1980.

[15] M. Romero, A. Lepage, and B. Lille, "Computational thinking development through creative programming in higher education," Int. J. Educ. Technol. High. Educ., vol. 14, no. 1, p. 42, 2017. https://doi.org/10.1186/s41239-017-0080-z

[16] S. Nicholson, "(PDF) Peeking Behind the Locked Door:A Survey of Escape Room Facilities." 2015.

[17] M. Resnick et al., "Digital manipulatives: new toys to think with," in Proceedings of the SIGCHI conference on Human factors in computing systems, 1998, pp. 281-287. https://doi.org/10.1145/274644.274684

[18] S. Nicholson, "Exploring Gamification Techniques for Classroom Management," p. 7, 2013.

[19] C. Obikwelu and J. C. Read, "The Serious Game Constructivist Framework for Children's Learning | Elsevier Enhanced Reader," 2012. [Online]. Available: https://reader.elsevier.com/reader/sd/pii/S1877050912008174?token=7E3B3840CDB9F237 
2D611DFA7A6F23B1F4454EF18F8E547091816645291FCC89F62D0F3B2F2BB958D9C0 9980B7A20C95. [Accessed: 18-Jul-2019].

[20] S. Nicholson, "Creating Engaging Escape Rooms for the Classroom," Child. Educ., vol. 94, no. 1, pp. 44-49, 2018. https://doi.org/10.1080/00094056.2018.1420363

[21] D. W. Johnson and R. T. Johnson, Cooperation and competition: Theory and research. Edina, MN, US: Interaction Book Company, 1989.

[22] M. Berland and V. R. Lee, "Collaborative strategic board games as a site for distributed computational thinking," Int. J. Game-Based Learn. IJGBL, vol. 1, no. 2, pp. 65-81, 2011. https://doi.org/10.4018/ijgbl.2011040105

[23] P. Apostolellis, M. Stewart, C. Frisina, and D. Kafura, "RaBit EscAPE: a board game for computational thinking," in Proceedings of the 2014 conference on Interaction design and children, 2014, pp. 349-352. https://doi.org/10.1145/2593968.2610489

[24] D. Wang, T. Wang, and Z. Liu, "A Tangible Programming Tool for Children to Cultivate Computational Thinking," The Scientific World Journal, 2014. [Online]. Available: https://www.hindawi.com/journals/tswj/2014/428080/abs/. [Accessed: 17-Apr-2019]. https://doi.org/10.1155/2014/428080

[25] M. Horn, "TopCodes: Tangible Object Placement Codes," 2012. [Online]. Available: http://users.eecs.northwestern.edu/ mhorn/topcodes/. [Accessed: 23-Sep-2019].

[26] C. Kazimoglu, M. Kiernan, L. Bacon, and L. MacKinnon, "Learning Programming at the Computational Thinking Level via Digital Game-Play," Procedia Comput. Sci., vol. 9, pp. 522-531, 2012. https://doi.org/10.1016/j.procs.2012.04.056

[27] C. M. Steiner, M. D. Kickmeier-Rust, and D. Albert, "MAKING SENSE OF GAME-BASED USER DATA: LEARNING ANALYTICS IN APPLIED GAMES," p. 4, 2015.

[28] S. Arnab et al., "Mapping learning and game mechanics for serious games analysis," Br. J. Educ. Technol., vol. 46, no. 2, pp. 391-411, 2015. https://doi.org/10.1111/bjet.12113

[29] K. Tsarava, K. Moeller, N. Pinkwart, M. Butz, U. Trautwein, and M. Ninaus, "Training Computational Thinking: Game-Based Unplugged and Plugged-in Activities in Primary School," in European Conference on Games Based Learning, 2017, pp. 687-695.

[30] T. Y. Lee, M. L. Mauriello, J. Ahn, and B. B. Bederson, "CTArcade: Computational thinking with games in school age children," Int. J. Child-Comput. Interact., vol. 2, no. 1, pp. 26-33, 2014. https://doi.org/10.1016/j.ijcci.2014.06.003

[31] A. Chaffin, K. Doran, D. Hicks, and T. Barnes, "Experimental evaluation of teaching recursion in a video game," in Proceedings of the 2009 ACM SIGGRAPH Symposium on Video Games - Sandbox '09, New Orleans, Louisiana, 2009, p. 79. https://doi.org/10.1145/1581073.1581086

[32] N. Brown, W. Darby, and H. Coronel, "An Escape Room as a Simulation Teaching Strategy," Clin. Simul. Nurs., vol. 30, pp. 1-6, 2019. https://doi.org/10.1016/j.ecns.2019.02.002

[33] S. Clarke, D. Peel, S. Arnab, L. Morini, H. Keegan, and O. Wood, "(PDF) EscapED: A Framework for Creating Educational Escape Rooms and Interactive Games to For Higher/Further Education." 2017. https://doi.org/10.17083/ijsg.v4i3.180

[34] C. Borrego, C. Fernández, I. Blanes, and S. Robles, "Room escape at class: Escape games activities to facilitate the motivation and learning in computer science," JOTSE, vol. 7, no. 2, pp. 162-171, 2017. https://doi.org/10.3926/jotse.247

[35] S. Tarkan et al., "Toque: designing a cooking-based programming language for and with children," in Proceedings of the SIGCHI conference on human factors in computing systems, 2010, pp. 2417-2426. https://doi.org/10.1145/1753326.1753692

[36] N. R. Council, Report of a Workshop on the Scope and Nature of Computational Thinking. 2010.

[37] S. Frey, "Webby's Challenge: A Coding Escape Room," Inverse Genius, 2018. [Online]. Available: http://www.inversegenius.com/gsl-blog/2018/5/1/webbys-challenge-a-codingescape-room. [Accessed: 06-May-2019].

[38] "Escape Code - Coding Adventure on Steam." [Online]. Available: https://store.steampowered.com/app/842350/Escape_Code_Coding_Adventure/. [Accessed: 06-May-2019].

[39] "WELCOME TO BREAKOUT EDU," Breakout EDU. [Online]. Available: https://www.breakoutedu.com/welcome. [Accessed: 16-Apr-2019].

[40] S. Werneburg, S. Manske, and H. U. Hoppe, "ctGameStudio - A Game-Based Learning Environment to Foster Computational Thinking," p. 10, 2018.

[41] K. Brennan and M. Resnick, "New frameworks for studying and assessing the development of computational thinking," p. 25, 2012. 
pag. 18

[42] S. Grover and S. Basu, "Measuring Student Learning in Introductory Block-Based Programming: Examining Misconceptions of Loops, Variables, and Boolean Logic." 2017. https://doi.org/10.1145/3017680.3017723

[43] J. Moreno-León, G. Robles, and M. Román-González, "Dr. Scratch: Automatic analysis of scratch projects to assess and foster computational thinking," RED Rev. Educ. Distancia, no. 46, pp. 1-23, 2015.

[44] M. Román-González, J.-C. Pérez-González, and C. Jiménez-Fernández, "Which cognitive abilities underlie computational thinking? Criterion validity of the Computational Thinking Test," Comput. Hum. Behav., vol. 72, pp. 678-691, 2017. https://doi.org/10.1016/j.chb.2016.08.047 